\title{
Microwave photoassisted dissipation and supercurrent of a phase-biased graphene-superconductor ring
}

\author{
Ziwei Dou $\odot,{ }^{1}$ Taro Wakamura, ${ }^{1}$ Pauli Virtanen $\odot,{ }^{2}$ Nian-Jheng Wu ${ }^{1,3}$ Richard Deblock $\odot,{ }^{1}$ Sandrine Autier-Laurent, \\ Kenji Watanabe $\odot,{ }^{4}$ Takashi Taniguchi, ${ }^{4}$ Sophie Guéron, ${ }^{1}$ Hélène Bouchiat, ${ }^{1}$ and Meydi Ferrier ${ }^{1}$ \\ ${ }^{1}$ Université Paris-Saclay, CNRS, Laboratoire de Physique des Solides, 91405 Orsay, France \\ ${ }^{2}$ Department of Physics and Nanoscience Center, University of Jyväskylä, Jyväskylä, Finland \\ ${ }^{3}$ Université Paris-Saclay, CNRS, Institut des Sciences Moléculaires d'Orsay, 91405 Orsay, France \\ ${ }^{4}$ National Institute for Materials Science, Tsukuba, Japan
}

(Received 21 November 2020; accepted 21 May 2021; published 8 July 2021)

\begin{abstract}
Irradiating normal-superconducting junctions with microwave photons produce spectacular effects, such as Shapiro steps and photoinduced modifications of the dc supercurrent. Moreover, microwave irradiation can also have other, hitherto unexplored consequences, such as a photoassisted dissipation which is phase dependent. Here we present a finite-frequency measurement of both the dissipation and the supercurrent of a phase-biased graphene-superconductor junction in response to microwave photons. We find that, while the supercurrent response is well described by existing theory, the dissipation exhibits unexpected effects which need new theoretical elucidation. Especially with high frequency photons, the dissipation is enhanced at phase zero, where it is minimum without irradiation. We attribute this enhancement to Andreev level transitions, made possible by microwave-induced nonequilibrium population of Andreev bound states. Our results demonstrate that dissipation is a more sensitive probe of microwave photons than is the supercurrent, and reveal the potential of measuring dissipation to improve superconducting photodetectors and investigate photoassisted physics in hybrid superconducting systems.
\end{abstract}

DOI: 10.1103/PhysRevResearch.3.L032009

Superconducting junctions and the related ac and dc Josephson effects are at the heart of a wide range of investigations, such as topological superconductivity [1,2], ultrasensitive photodetectors [3,4], and quantum information devices [5], many of which exploit the interaction between the junctions and microwave photons. However, several experiments revolving around the ac Josephson effect, such as those measuring current-phase relations (CPRs) modified by microwave irradiation [6], or others searching for the missing Shapiro steps in topological Josephson junctions [7], have demonstrated the need for a more thorough understanding of the microwave photo-assisted dynamics of normal-superconducting (NS) junctions. In particular, experiments that go beyond the measurement of the sole dc supercurrent are bound to give more insight into the dynamics of Andreev bound states (ABSs) carrying the supercurrent $[8,9]$. To achieve this goal, we perform finite frequency measurement of a microwave-irradiated, phase-biased NS ring. In addition to the dissipationless response (related to the dc supercurrent), we report in this Letter the less explored dissipative response.

By embedding the NS ring in a superconducting rf resonator, the ac magnetic field introduces an ac component

Published by the American Physical Society under the terms of the Creative Commons Attribution 4.0 International license. Further distribution of this work must maintain attribution to the author(s) and the published article's title, journal citation, and DOI. $(\delta \varphi)$ to the dc superconducting phase $(\varphi)$ controlled by the magnetic flux. The instantaneous current response is simply proportional to the phase derivative of the dissipationless CPR. On the other hand, finite-time relaxation of ABSs towards equilibrium causes delay in the current response and consequently a dissipation appears [8]. Such dissipation has previously revealed the relaxation of thermally excited ABSs via inelastic scattering, which can distinguish between topological and nontopological Andreev level crossings [8,9]. Here we extend this measurement to the phase-dependent, photoassisted dissipation and supercurrent in a wide range of frequency and power of a graphene-superconductor ring in the long diffusive regime. We demonstrate the higher sensitivity of the dissipative response to microwave irradiation which drives the system out of equilibrium, especially when the irradiation frequency exceeds the minigap of the system.

Graphene forms an ideal normal weak link due to its low density of states. This leads to a much smaller critical current than metallic weak links [10], and therefore reduces screening effects. This in turn allows for accurate measurement without distortion over a complete phase period even at low temperature, which is not accessible in other conventional metallic NS junctions $[8,11]$.

Figure 1(a) shows the scanning electron microscopy (SEM) images of the device. The left image shows a section of the resonator (the meander lines) made by e-beam lithography and sputtered molybdenum-rhenium (MoRe) on the undoped silicon substrate. The boron-nitride/graphene/boron-nitride $(\mathrm{BN} / \mathrm{G} / \mathrm{BN})$ stack is fabricated using exfoliated flakes and is then connected to MoRe via side contacts [12]. The details 


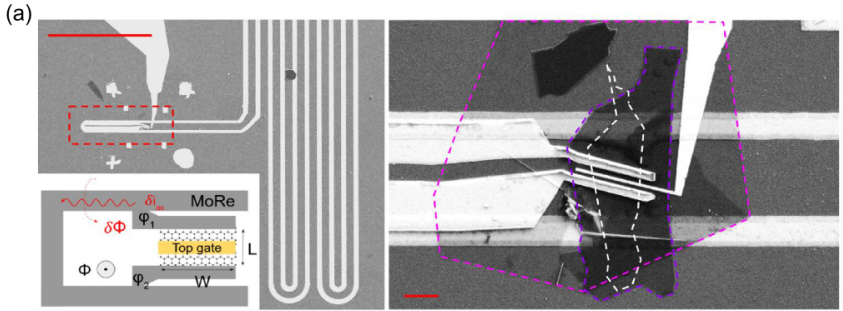

(b)

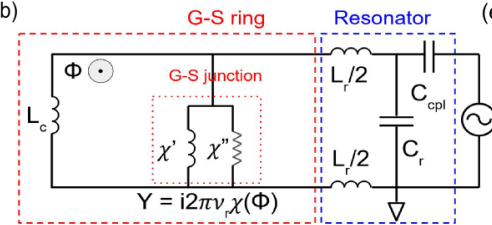

(e)
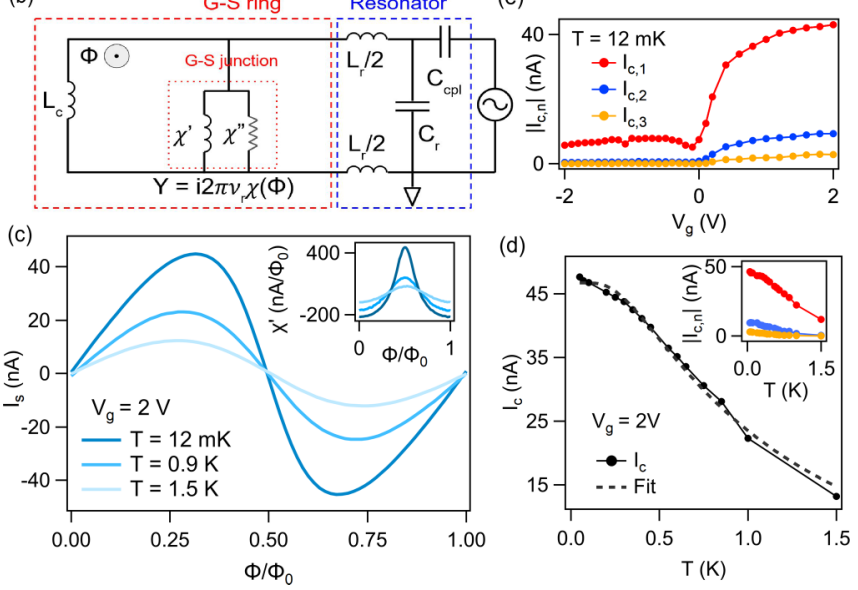

FIG. 1. CPRs without microwave irradiation: (a) SEM images of the device. Left: a section of the MoRe resonator. Scale bar: $100 \mu \mathrm{m}$. Inset: schematic of the graphene-superconductor ring. Right: zoomed-in image of the SGS junction. Scale bar: $3 \mu \mathrm{m}$. Purple and magenta dashed outlines: top and bottom BN. White dashed outline: graphene. (b) Circuit diagram. For explanation, see text. (c) CPRs at $V_{g}=2 \mathrm{~V}$, and $T=12 \mathrm{mK}, 0.9 \mathrm{~K}$, and $1.5 \mathrm{~K}$. Inset: $\chi^{\prime}(\Phi)$. (d) The critical current $I_{c}$ versus $T$ at $V_{g}=2 \mathrm{~V}$. Dashed line: fitting to diffusive model with $E_{T h}=34 \mu \mathrm{eV}$. Inset: the amplitudes of the first three Fourier coefficients of the CPRs $\left|I_{c, n}\right|(n=1,2,3)$ versus $T$. (e) $\left|I_{c, n}\right|(n=1,2,3)$ versus $V_{g}$ at $T=12 \mathrm{mK}$.

of the fabrication are given in [12-15]. The junction width is $W=5 \mu \mathrm{m}$ and the length $L=950 \mathrm{~nm}$. The Ti/Au top gate covers $330 \mathrm{~nm}$ of the total graphene length. The junction is probed by $\mathrm{rf}$ reflectometry. In Fig. 1(b), the resonator is designed to have $L_{r}=40 \mathrm{nH}$. $C_{r}=180 \mathrm{pF}$ is chosen to give a resonance frequency $v_{r}=60 \mathrm{MHz}$. The loop at the end of the superconducting lines provides a coupling inductance $L_{c}=355 \mathrm{pH}$. To maintain sufficiently high quality factor $Q$, the resonator is coupled to the rf coaxial cables through a coupling capacitor $C_{c p l}=5.6 \mathrm{pF}$. At $T=12 \mathrm{mK}, Q \sim 300$. The dc flux $\Phi$ (or the dc phase $\varphi=\varphi_{1}-\varphi_{2}=2 \pi \Phi / \Phi_{0}$ ) is set by the dc magnetic field through the area defined by the ring, and is added to the ac flux $\delta \Phi$ inducing the ac current $\delta i_{a c}$. The ac susceptibility of the junction is thus defined as $\chi=\delta i_{a c} / \delta \Phi$ [8]. The rf power is heavily attenuated so that $\delta \Phi \ll \Phi_{0}$ and $\chi$ does not depend on the rf power. At finite frequency, $\chi=\chi^{\prime}+i \chi^{\prime \prime}$ is complex and the complex admittance $Y=i 2 \pi v_{r} \chi$ thus can be modeled as an effective inductance and conductance in parallel [Fig. 1(b)]. By circuit theory, the total admittance of the ring $Y_{\text {tot }}=Y+$ $1 /\left(i 2 \pi v_{r} L_{c}\right)$ determines both the resonance frequency $v_{r}$ and the quality factor $Q$ of the resonator, and on resonance the real (dissipationless) and imaginary (dissipative) parts $\chi^{\prime}$ and $\chi^{\prime \prime}$ are linked to $v_{r}$ and $Q$ via $\chi^{\prime}=-2\left(L_{r} / L_{c}^{2}\right)\left(\delta v_{r} / v_{r}\right)$ and $\chi^{\prime \prime}=\left(L_{r} / L_{c}^{2}\right) \delta(1 / Q)[8]$. As $\Phi$ is swept, $\delta v_{r}$ and $\delta(1 / Q)$ are simultaneously measured using a phase-locked feedback loop which maintains the resonator on resonance [8,16,17]. At sufficiently low frequency $60 \mathrm{MHz}$ where $\chi^{\prime \prime} / \chi^{\prime} \ll 1$ [13], $\chi^{\prime}(\Phi) \approx \partial I_{s} / \partial \Phi=\left(2 \pi / \Phi_{0}\right)\left(\partial I_{s} / \partial \varphi\right)$, where $I_{s}$ is the supercurrent [8]. Thus, integrating the measured $\chi^{\prime}(\Phi)$ yields $I_{s}(\varphi)$, the same as the conventionally measured CPR by, for instance, a de SQUID.

Figure 1(c) displays the CPRs $I_{s}(\Phi)$ at $T=12 \mathrm{mK}, 0.9 \mathrm{~K}$, and $1.5 \mathrm{~K}\left[\chi^{\prime}(\Phi)\right.$ in inset]. The critical current at base temperature is $I_{c}=50 \mathrm{nA}$. Indeed, $\beta=2 \pi L_{c} I_{c} / \Phi_{0}=0.03 \ll 1$; thus the distortion in $\chi$ by the flux-dependent screening effect is negligible $[8,11]$. We also extract the first three Fourier coefficients $I_{c, n}(n=1,2,3)$ from the CPRs, where $I_{s}(\varphi) \approx$ $I_{c, 1} \sin (\varphi)+I_{c, 2} \sin (2 \varphi)+I_{c, 3} \sin (3 \varphi) . I_{c}(T)$ and $\left|I_{c, n}\right|$ are plotted in Fig. 1(d). Note $I_{c, 2}$ is negative and $\left|I_{c, 2}\right|=-I_{c, 2}$. At $T=12 \mathrm{mK},\left|I_{c, 2}\right|$ and $\left|I_{c, 3}\right|$ have non-negligible values, consistent with the skewed CPR in Fig. 1(c), while at $T=1.5$ $\mathrm{K}$ only $\left|I_{c, 1}\right|$ is dominant, meaning the CPR is sinusoidal. This is similar to previous measurement of the graphene junction $[18,19]$. Assuming a long diffusive junction model at low temperature, we can fit $I_{c}(T)$ by $I_{c}=\left(7.7 E_{T h} / e R\right)[1-$ $\left.1.3 \exp \left(-7.7 E_{T h} / 3.2 k_{B} T\right)\right]$, where Thouless energy $E_{T h}$ and normal resistance $R$ are two fitting parameters [20,21]. The fitting yields $R=5.6 \mathrm{k} \Omega$ and $E_{T h}=34 \mu \mathrm{eV}$ (equivalently $400 \mathrm{mK}$ or $8 \mathrm{GHz}$ ). Since $E_{T h}=\hbar v_{F} l_{e} / 2 L^{2}$ [20], the meanfree path is $l_{e} \approx 100 \mathrm{~nm}$. The minigap $E_{g}=2 \times 3.1 E_{T h}=$ $206 \mu \mathrm{eV}$ (or $46 \mathrm{GHz}$ ) [22] is higher than the irradiation frequency accessible in later experiment. However, the above estimate assumes perfect interface and strong disorder. By numeric simulation [13,23-25], we show that $2 \times 3.1 E_{T h}$ overestimates the minigap in junction with imperfect contacts and weak disorder, which is plausible in the BN-encapsulated graphene sample. The actual $E_{g}$ may thus fall within the frequency range in the experiment. In the Supplemental Material $[13,19,26-28]$, we also justify that our junction does not agree with the ballistic model.

Figure 1(e) shows $\left|I_{c, n}\right|$ versus $V_{g}$ at $T=12 \mathrm{mK}$. At $V_{g}=2$ $\mathrm{V}$, the supercurrent is almost saturated at $44 \mathrm{nA}$, smaller than in previous studies $[18,29,30]$ possibly because of insufficiently filtered radiation from environment and the higher normal resistance of graphene in the nongated regions. Meanwhile, $\left|I_{c, 1}\right| \sim 5\left|I_{c, 2}\right|$ at $V_{g}=2 \mathrm{~V}$, similar to a uniformly gated sample [18], meaning the transparency between the MoRe and graphene is still enough to preserve $I_{c, 2}$. For negative $V_{g},\left|I_{c, 1}\right|$ is much reduced and $\left|I_{c, 2}\right|,\left|I_{c, 3}\right|$ become negligible, consistent with the formation of pn junctions $[18,30]$. Throughout the paper we focus on the high electron-doping regime with $V_{g}=2 \mathrm{~V}$ for the highest susceptibility signal.

After exploring the junction without microwave irradiation and finding the CPRs agree with previous experiments $[18,19,30]$, we now present the data with irradiation. In Figs. 2(a) and 2(b), the CPRs are measured at two irradiation frequencies $v=2 \mathrm{GHz}$ and $v=19 \mathrm{GHz}$, respectively. The power noted in the figure is converted to the normalized power $s=\alpha e V_{p k} / h v\left(V_{p k}\right.$ is the peak voltage at the source and $\alpha$ includes the attenuation factor from source to junction). In both figures, a sign reversal of the supercurrent occurs as the irradiation power increases. However, for $v=2 \mathrm{GHz}$, a strong second harmonic (halved periodicity) is observed 

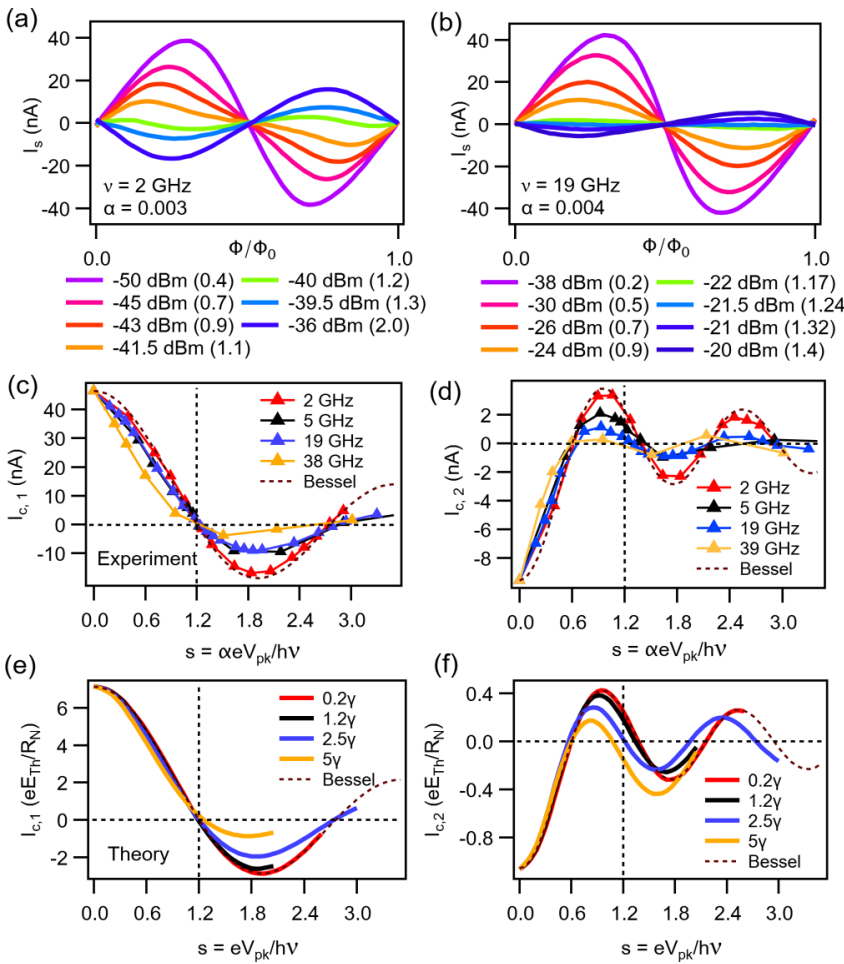

(f)

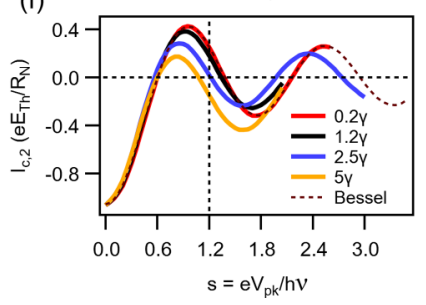

FIG. 2. Effects of microwave irradiation on CPR Fourier coefficients: (a) CPRs with irradiation $v=2 \mathrm{GHz}$. The irradiation power is converted to the normalized value $s=\alpha e V_{p k} / h v$ in parentheses (see text). The sign reversal of $I_{s}$ is observed at $s \sim 1.2$ with halved periodicity. (b) CPRs with $v=19 \mathrm{GHz}$. The sign reversal of $I_{s}$ is also seen at $s \sim 1.2$, but without halved periodicity. (c), (d) $I_{c, 1}(s)$ and $I_{c, 2}(s)$. All data are taken at $V_{g}=2 \mathrm{~V}$ and $T=12 \mathrm{mK}$. (e), (f) Calculated $I_{c, 1}(s)$ and $I_{c, 2}(s)$ from Usadel equations with finite relaxation rate $\gamma=1.2 E_{T h} . k_{B} T=0.004 \gamma . \Delta / E_{T h}=50$. Bessel functions $I_{c, 1}(0) J(2 s)$ and $I_{c, 2}(0) J(4 s)$ are plotted for (c), (e) and (d), (f), respectively (dashed lines). $J$ is the zeroth order Bessel function of the first kind. $I_{c, 1}(0)=44 \mathrm{nA}$, and $I_{c, 2}(0)=-9 \mathrm{nA}$ are the nonirradiated values from Fig. 1.

during the reversal, while almost no phase dependence is seen for $v=19 \mathrm{GHz}$ in a similar situation. In Figs. 2(c) and 2(d) we plot the power dependence of the first two Fourier coefficients of the CPRs for $v$ between $2 \mathrm{GHz}$ and $39 \mathrm{GHz}$. At low irradiation frequency, the junction shows the adiabatic ac Josephson effect [31], where ABSs follow instantaneously the oscillating $\delta \varphi$ and $I_{c, n}(s)$ shows the Bessel function dependence. The experimental $\alpha$ is hard to calibrate accurately, and for each $I_{c, 1}(s), \alpha$ is chosen such that its first zero coincides with $s=1.2$ (the first zero of the Bessel function). The same $\alpha$ is then used for $I_{c, 2}(s)$ of the respective frequency. The $2 \mathrm{GHz}$ data agrees well with the Bessel function, indicating the microwave drive is adiabatic [32]. It also shows that the electronic temperature is not significantly increased by irradiation. For higher frequencies, the agreement is less satisfactory. In particular, $I_{c, 2}$ at $s=1.2$ decreases for higher $v$, which is consistent with a much weaker second harmonic (halved periodicity) in the CPR for $v=19 \mathrm{GHz}$ in Fig. 2(b) compared to $v=2 \mathrm{GHz}$ in Fig. 2(a). Using time-dependent Usadel equations incorporating a finite inelastic scattering rate $\gamma$ [32], we calculate $I_{c, 1}(s)$ and $I_{c, 2}(s)$ at low temperature and (a)
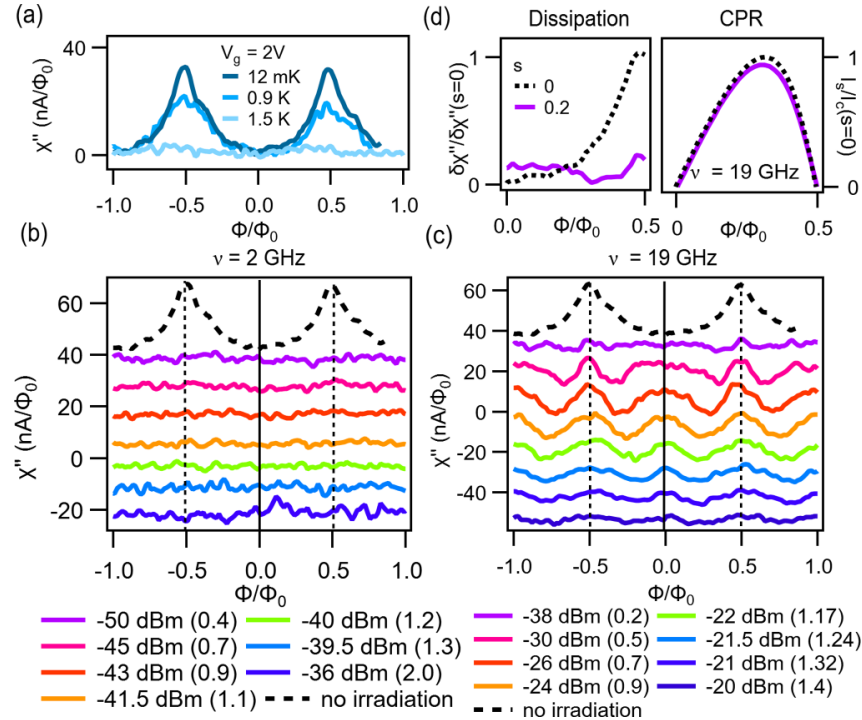

FIG. 3. Effects of microwave irradiation on the dissipative part of the susceptibility: (a) $\chi^{\prime \prime}(\Phi)$ without irradiation at $T=12 \mathrm{mK}$, $0.9 \mathrm{~K}$, and $1.5 \mathrm{~K}$, taken simultaneously as $\chi^{\prime}(\Phi)$ leading to the CPRs in Fig. 1(c). (b) $\chi^{\prime \prime}(\Phi)$ taken simultaneously as the CPRs in Fig. 2(a). The irradiation frequency $v=2 \mathrm{GHz}$. The irradiation power is converted to the normalized value $s$ as in Fig. 2. The black dashed line is the $\chi^{\prime \prime}(\Phi)$ without irradiation. $\chi^{\prime \prime}(\Phi)$ shows no significant $\Phi$ dependence under irradiation. (c) $\chi^{\prime \prime}(\Phi)$ taken simultaneously as the CPRs in Fig. 2(b). $v=19 \mathrm{GHz} . \chi^{\prime \prime}(\Phi)$ changes from $2 \pi$ periodic with no irradiation to $\pi$ periodic under higher irradiation power. (d) Left: $\delta \chi^{\prime \prime}$ normalized by unirradiated value $(s=0)$. Right: $I_{s}$ in Fig. 2(b) normalized by critical current at $s=0 . V_{g}=2 \mathrm{~V}$ and $T=12 \mathrm{mK}$ for (b), (c), and (d). The $\chi^{\prime \prime}$ curves are shifted vertically for clarity.

$v$ lower and higher than $\gamma$. The results are plotted in Figs. 2(e) and 2(f). At $v=0.2 \gamma, I_{c, 1}(s)$ and $I_{c, 2}(s)$ follow the Bessel function as expected, while for $v>\gamma$ they show deviation in qualitative agreement to Figs. 2(c) and 2(d). Comparing the theory $(1.2 \gamma$ and $2.5 \gamma$ curves $)$ with the experiment $(5 \mathrm{GHz}$ and $19 \mathrm{GHz}$ curves), the experimental $\gamma$ can be estimated as between $5 / 1.2 \mathrm{GHz}=4 \mathrm{GHz}$ and $19 / 2.5 \mathrm{GHz}=8 \mathrm{GHz}$. Thus $h \gamma \lesssim E_{T h}$, reasonable for SNS junctions [8].

The finite $v_{r} / \gamma$ gives rise to a nonzero dissipation $\chi^{\prime \prime}$ [8]. Figure 3 (a) shows $\chi^{\prime \prime}(\Phi)$ taken simultaneously as the CPRs in Fig. 1(c) without irradiation. $\chi^{\prime \prime}(\Phi)$ peaks at $0.5 \Phi_{0}$ and its height decreases with temperature. This higher dissipation is a result of the minigap closing which allows more excitation-relaxation events between Andreev states [8]. At low temperature, $\delta \chi^{\prime \prime} / \delta \chi^{\prime} \sim 20$ [here $\delta \chi=\chi\left(0.5 \Phi_{0}\right)-$ $\chi(0)]$ is indeed similar to $v_{r} / \gamma$ estimated independently from CPRs, as discussed in [13]. Figures 3(b) and 3(c) show $\chi^{\prime \prime}(\Phi)$ under irradiation taken simultaneously as the CPRs in Figs. 2(a) and 2(b). For both $v=2 \mathrm{GHz}$ and $19 \mathrm{GHz}$, the small irradiation power flattens $\chi^{\prime \prime}(\Phi)$. More data in [13] shows a gradual diminution in $\delta \chi^{\prime \prime}$ for smaller power. As illustrated in Fig. 3(d), $\delta \chi^{\prime \prime}(s=0.2)$ is decreased by $80 \%$ of the unirradiated $\delta \chi^{\prime \prime}(s=0)$, whereas the CPR is almost unaffected, demonstrating the much higher power sensitivity of $\delta \chi^{\prime \prime}$ than CPR. In the Supplemental Material [13], we show that phase oscillation from the ac Josephson effects can 
partly explain the flattened $\chi^{\prime \prime}(\varphi)$ at low $s$. At high irradiation power, the dissipation response is drastically different for $v=19 \mathrm{GHz}$ : instead of the flat $\chi^{\prime \prime}(\Phi)$ in Fig. 3(c), an additional lobe appears in Fig. 3(d) around flux zero and the peak at $0.5 \Phi_{0}$ becomes pronounced again, which turns $\chi^{\prime \prime}(\Phi)$ into a function quasiperiodic in $\Phi_{0} / 2$. This emergence of an enhanced dissipation at flux zero under high irradiation frequency and power is a key observation of this Letter and requires an explanation.

While $\chi^{\prime \prime}(\Phi)$ with nonzero $v_{r}$ can be calculated by a similar Usadel equation approach [33] to that in Fig. 2 for the CPRs in the de limit, it is not straightforward to include both the low-frequency ac flux and the high-frequency irradiation. In order to explain Fig. 3(c), we thus turn to the Kubo formalism, which describes well the $\chi^{\prime \prime}(\Phi)$ of diffusive junctions without irradiation $[8,34,35]$. In our junction, the rf frequency used for susceptibility measurement $v_{r}$ is much smaller than the inelastic scattering rate $\gamma$; thus $\chi^{\prime \prime}$ can be approximated by [13]

$$
\chi^{\prime \prime} \approx-\operatorname{Im}\left[\sum_{n, m \neq n}\left|J_{n m}\right|^{2} \frac{f_{n}-f_{m}}{\epsilon_{n}-\epsilon_{m}} \frac{i h v_{r}}{i\left(\epsilon_{n}-\epsilon_{m}-h v_{r}\right)+h \gamma}\right],
$$

where $J_{n m}=\left(e \hbar / m_{e} i\right)\langle n|\nabla| m\rangle$ is the off-diagonal element of the current operator and $\epsilon_{n}$ is the $n$th Andreev level. The effect of the microwave irradiation is included phenomenologically in the distribution function:

$$
f_{n}=(1-\sigma) f_{F D}\left(\epsilon_{n}\right)+\sigma / 2\left[f_{F D}\left(\epsilon_{n}+h \nu\right)+f_{F D}\left(\epsilon_{n}-h \nu\right)\right],
$$

where $v$ is the irradiation frequency and $\sigma$ stands for the irradiation power. Without irradiation $(\sigma=0), f_{n}$ follows the Fermi-Dirac distribution $f_{F D}\left(\epsilon_{n}\right)=1 /\left[1+\exp \left(\epsilon_{n} / k_{B} T\right)\right]$. With the irradiation, the change in distribution function from the equilibrium one $\delta f\left(\epsilon_{n}\right)=f_{n}-f_{F D}$ shown in Fig. 4(a) qualitatively reproduces the results based on the timedependent Green's function approach near $\varphi=0$ [32,36,37]: the Andreev state occupation is depleted (enhanced) within $h v$ below (above) the minigap by irradiation photons. The total $\chi^{\prime \prime}$ can thus be rewritten as $\chi^{\prime \prime}=(1-\sigma) \chi_{0}^{\prime \prime}+\sigma / 2\left[\chi_{+h v}^{\prime \prime}+\right.$ $\left.\chi_{-h v}^{\prime \prime}\right]$, where $\chi_{0}^{\prime \prime}$ and $\chi_{ \pm h v}^{\prime \prime}$ are calculated with Eq. (1) using $f_{F D}(E)$ and $f_{F D}(E \pm h v)$, respectively. We numerically calculate these terms in a diffusive SNS junction using the tight-binding method [23,35]. See details in [13]. The Andreev spectrum near $E=0$ is plotted in Fig. 4(a) showing a minigap. Figure 4(b) displays two examples $\left|J_{1,-1}\right|^{2}$ and $\left|J_{23,44}\right|^{2}$ whose corresponding $\left(f_{n}-f_{m}\right) /\left(\epsilon_{n}-\epsilon_{m}\right)$ are nonzero. They represent "interband" transition (red arrow) and "intraband" transition (blue arrow), respectively. As $\sigma$ goes from 0 to $0.8, \chi^{\prime \prime}(\varphi)$ evolves from a $2 \pi$-periodic function to a quasi- $\pi$-periodic function, which is the key feature in Fig. 3(c). By comparing Figs. 4(c) and 4(d), the rising lobe at phase zero is attributed to the nonequilibrium terms $\chi_{ \pm h v}^{\prime \prime}$ and can be understood as follows: at low temperature, the equilibrium $f_{F D}$ is close to a step function; thus only interband terms $\left|J_{n,-n}\right|^{2}$ contribute to Eq. (6). Since $\left|J_{n,-n}\right|^{2}$ generally peaks at $\pi$ (e.g., $\left|J_{1,-1}\right|^{2}$ ) [35], $\chi_{0}^{\prime \prime}(\pi)$ is also high. Meanwhile, under irradiation with $h v>E_{g}$, the distribution function is modified
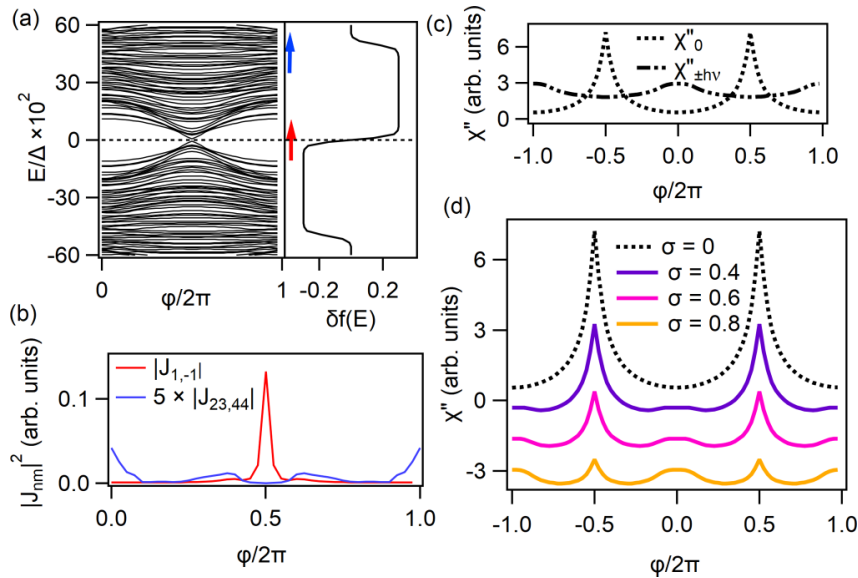

FIG. 4. Calculation of $\chi^{\prime \prime}(\Phi)$ with nonequilibrium distribution function: (a) Andreev spectrum of a long diffusive system displaying a minigap $E_{g} \sim 0.2 \Delta . \Delta$ is the superconducting pairing potential. Side plot: distribution function change $\delta f(E)$ due to irradiation (see text). (b) The interband term $\left|J_{1,-1}\right|^{2}$ (red) and the intraband term $\left|J_{23,44}\right|^{2}$ (blue). The corresponding transitions are marked by the arrows in the same colors in (a). (c) $\chi_{0}^{\prime \prime}(\varphi)$ calculated with $f_{F D}(E)$ (dashed line) and $\chi_{ \pm h v}^{\prime \prime}(\varphi)$ calculated with $f_{F D}(E \pm h v)$ (dashed-dotted line). (d) Total $\chi^{\prime \prime}(\Phi) . \sigma$ stands for the irradiation power ( $\sigma=0$ being no irradiation). Curves are offset for clarity. Similar to experiment, $k_{B} T=h v_{r}=0.01 \Delta \ll E_{g}$, relaxation rate $h \gamma=0.15 \Delta \sim 0.5 E_{g}$, and irradiation frequency $h v=0.5 \Delta \sim 2 E_{g}$. System size: $W \times L=50 \times 30$ sites.

and states near the minigap are partially occupied, enabling more intraband transitions. The intraband terms $\left|J_{n m}\right|^{2}$, which in general have higher magnitudes around phase zero (see $\left|J_{23,44}\right|^{2}$ ), produce an enhanced $\chi_{ \pm h v}^{\prime \prime}(0)$. The same simulation with $h v<E_{g}$ only shows the $2 \pi$-periodic $\chi^{\prime \prime}$.

In conclusion, we simultaneously measure the phasedependent dissipation and supercurrent via the ac susceptibility of a graphene/superconductor junction, subject to microwave photons. While the photoassisted CPRs for the whole range of irradiation frequency and power agree qualitatively with the existing dc theory based on the semiclassical Usadel equation, the photoassisted dissipation exhibits distinctive effects which require new explanations. First, we observe a higher sensitivity of dissipation than supercurrent to microwave power at various frequencies. Second, particularly with high frequency photons, the dissipation is enhanced at phase zero, where it is minimum without microwave. Using Kubo formalism, we explain this characteristic response as a signature of microwave-induced nonequilibrium population of ABSs, which enables intraband transitions forbidden at equilibrium. Our results suggest the potential role of so-far unexploited dissipation of an SNS junction operating in microwave: it may inspire further improvements of ultrasensitive microwave photodetectors based on superconducting devices $[3,4]$, as well as shed new light on the photoassisted Andreev state dynamics in novel hybrid superconducting systems $[38,39]$.

We acknowledge insightful discussion with T. T. Heikkilä, M. Aprili, and J. Estève. We also acknowledge financial 
support from ERC Ballistop (ERC Grant No. 833350), Japan Society for the Promotion of Science (Grant No. 2017-684),
LabEx PALM (Grant No. ANR-10-LABX-0039-PALM), and ANR JETS (Grant No. ANR-16-CE30-0029-01).
[1] C. Li, J. de Boer, B. de Ronde, S. Ramankutty, E. van Heumen, Y. Huang, A. de Visser, A. Golubov, M. Golden, and A. Brinkman, $4 \pi$-periodic Andreev bound states in a Dirac semimetal, Nat. Mater. 17, 875 (2018).

[2] E. Bocquillon, R. Deacon, J. Wiedenmann, P. Leubner, T. Klapwijk, C. Brüne, K. Ishibashi, H. Buhmann, and L. Molenkamp, Gapless Andreev bound states in the quantum spin Hall insulator HgTe, Nat. Nanotechnol. 12, 137 (2017).

[3] G.-H. Lee, D. Efetov, W. Jung, L. Ranzani, E. Walsh, T. Ohki, T. Taniguchi, K. Watanabe, P. Kim, D. Englund, and K. Fong, Graphene-based Josephson junction microwave bolometer, Nature (London) 586, 42 (2020).

[4] R. Kokkoniemi, J.-P. Girard, D. Hazra, A. Laitinen, J. Govenius, R. E. Lake, I. Sallinen, V. Vesterinen, M. Partanen, J. Y. Tan, K. W. Chan, K. Y. Tan, P. Hakonen, and M. Möttönen, Bolometer operating at the threshold for circuit quantum electrodynamics, Nature (London) 586, 47 (2020).

[5] L. Casparis, M. R. Connolly, M. Kjaergaard, N. J. Pearson, A. Kringhøj, T. W. Larsen, F. Kuemmeth, T. Wang, C. Thomas, S. Gronin, G. C. Gardner, M. J. Manfra, C. M. Marcus, and K. D. Petersson, Superconducting gatemon qubit based on a proximitized two-dimensional electron gas, Nat. Nanotechnol. 13, 915 (2018).

[6] J. Basset, M. Kuzmanović, P. Virtanen, T. T. Heikkilä, J. Estève, J. Gabelli, C. Strunk, and M. Aprili, Nonadiabatic dynamics in strongly driven diffusive Josephson junctions, Phys. Rev. Research 1, 032009(R) (2019).

[7] M. C. Dartiailh, J. J. Cuozzo, B. H. Elfeky, W. Mayer, J. Yuan, K. S. Wickramasinghe, E. Rossi, and J. Shabani, Missing Shapiro steps in topologically trivial Josephson junction on InAs quantum well, Nat. Commun. 12, 78 (2021).

[8] B. Dassonneville, A. Murani, M. Ferrier, S. Guéron, and H. Bouchiat, Coherence-enhanced phase-dependent dissipation in long SNS Josephson junctions: Revealing Andreev bound state dynamics, Phys. Rev. B 97, 184505 (2018).

[9] A. Murani, B. Dassonneville, A. Kasumov, J. Basset, M. Ferrier, R. Deblock, S. Guéron, and H. Bouchiat, Microwave Signature of Topological Andreev Level Crossings in a Bismuth-Based Josephson Junction, Phys. Rev. Lett. 122, 076802 (2019).

[10] C. Li, S. Guéron, A. Chepelianskii, and H. Bouchiat, Full range of proximity effect probed with superconductor/graphene/superconductor junctions, Phys. Rev. B 94, 115405 (2016).

[11] M. Fuechsle, J. Bentner, D. A. Ryndyk, M. Reinwald, W. Wegscheider, and C. Strunk, Effect of Microwaves on the Current-Phase Relation of Superconductor-Normal-MetalSuperconductor Josephson Junctions, Phys. Rev. Lett. 102, 127001 (2009).

[12] L. Wang, I. Meric, P. Y. Huang, Q. Gao, Y. Gao, H. Tran, T. Taniguchi, K. Watanabe, L. M. Campos, D. A. Muller, J. Guo, P. Kim, J. Hone, K. L. Shepard, and C. R. Dean, One-dimensional electrical contact to a two-dimensional material, Science 342, 614 (2013).
[13] See Supplemental Material at http://link.aps.org/supplemental/ 10.1103/PhysRevResearch.3.L032009 for details of device fabrication, numerical simulation and more discussion.

[14] S. Thoms and D. S. Macintyre, Investigation of CSAR 62, a new resist for electron beam lithography, J. Vac. Sci. Technol. B 32, 06FJ01 (2014).

[15] A. Castellanos-Gomez, M. Buscema, R. Molenaar, V. Singh, L. Janssen, H. S. J. van der Zant, and G. A. Steele, Deterministic transfer of two-dimensional materials by all-dry viscoelastic stamping, 2D Mater. 1, 011002 (2014).

[16] F. Chiodi, M. Ferrier, K. Tikhonov, P. Virtanen, T. T. Heikkilä, M. Feigelman, S. Guéron, and H. Bouchiat, Probing the dynamics of Andreev states in a coherent normal/superconducting ring, Sci. Rep. 1, 3 (2011).

[17] B. Reulet, H. Bouchiat, and D. Mailly, Ac Conductance of Mesoscopic Rings (Springer Netherlands, Dordrecht, 1995), pp. 341-357.

[18] G. Nanda, J. L. Aguilera-Servin, P. Rakyta, A. Korményos, R. Kleiner, D. Koelle, K. Watanabe, T. Taniguchi, L. M. K. Vandersypen, and S. Goswami, Current-phase relation of ballistic graphene Josephson junctions, Nano Lett. 17, 3396 (2017).

[19] L. Bretheau, J. I.-J. Wang, R. Pisoni, K. Watanabe, T. Taniguchi, and P. Jarillo-Herrero, Tunnelling spectroscopy of Andreev states in graphene, Nat. Phys. 13, 756 (2017).

[20] P. Dubos, H. Courtois, B. Pannetier, F. K. Wilhelm, A. D. Zaikin, and G. Schön, Josephson critical current in a long mesoscopic S-N-S junction, Phys. Rev. B 63, 064502 (2001).

[21] The first numerical factor 7.7 is determined by $\Delta / E_{T h} \approx 20$. Here the superconducting gap of more is $\Delta=1.8 k_{B} T_{c}$, where $T_{c} \approx 6 \mathrm{~K}$. The second numeric factor 3.2 is universal..

[22] D. A. Ivanov, R. von Roten, and G. Blatter, Minigap in a long disordered SNS junction: Analytical results, Phys. Rev. B 66, 052507 (2002).

[23] C. W. Groth, M. Wimmer, A. R. Akhmerov, and X. Waintal, Kwant: a software package for quantum transport, New J. Phys. 16, 063065 (2014).

[24] W. Belzig, F. K. Wilhelm, C. Bruder, G. Schön, and A. D. Zaikin, Quasiclassical Green's function approach to mesoscopic superconductivity, Superlattices Microstruct. 25, 1251 (1999).

[25] K. K. Likharev, Superconducting weak links, Rev. Mod. Phys. 51, 101 (1979).

[26] J. Cayssol, T. Kontos, and G. Montambaux, Isolated hybrid normal/superconducting ring in a magnetic flux: From persistent current to Josephson current, Phys. Rev. B 67, 184508 (2003).

[27] D. Sticlet, B. Dóra, and J. Cayssol, Ballistic transport through irradiated graphene, Phys. Rev. B 88, 205401 (2013).

[28] D. Nikolić, W. Belzig, and J. C. Cuevas, Local density of states in clean two-dimensional superconductor-normal metal-superconductor heterostructures, Phys. Rev. Research 1, 033031 (2019). 
[29] C. T. Ke, I. V. Borzenets, A. W. Draelos, F. Amet, Y. Bomze, G. Jones, M. Craciun, S. Russo, M. Yamamoto, S. Tarucha, and G. Finkelstein, Critical current scaling in long diffusive graphene-based Josephson junctions, Nano Lett. 16, 4788 (2016).

[30] I. V. Borzenets, F. Amet, C. T. Ke, A. W. Draelos, M. T. Wei, A. Seredinski, K. Watanabe, T. Taniguchi, Y. Bomze, M. Yamamoto, S. Tarucha, and G. Finkelstein, Ballistic Graphene Josephson Junctions from the Short to the Long Junction Regimes, Phys. Rev. Lett. 117, 237002 (2016).

[31] M. Tinkham, Introduction to Superconductivity, Dover Books on Physics Series (Dover Publications, Mineola, NY, 2004).

[32] P. Virtanen, T. T. Heikkilä, F. S. Bergeret, and J. C. Cuevas, Theory of Microwave-Assisted Supercurrent in Diffusive SNS Junctions, Phys. Rev. Lett. 104, 247003 (2010).

[33] P. Virtanen, F. S. Bergeret, J. C. Cuevas, and T. T. Heikkilä, Linear ac response of diffusive SNS junctions, Phys. Rev. B 83, 144514 (2011).
[34] N. Trivedi and D. A. Browne, Mesoscopic ring in a magnetic field: Reactive and dissipative response, Phys. Rev. B 38, 9581 (1988).

[35] M. Ferrier, B. Dassonneville, S. Guéron, and H. Bouchiat, Phase-dependent Andreev spectrum in a diffusive SNS junction: Static and dynamic current response, Phys. Rev. B 88, 174505 (2013).

[36] U. Gunsenheimer and A. D. Zaikin, Ballistic transport in superconducting weak links in a microwave field, Europhys. Lett. 41, 195 (1998).

[37] M. Eschrig, Distribution functions in nonequilibrium theory of superconductivity and Andreev spectroscopy in unconventional superconductors, Phys. Rev. B 61, 9061 (2000).

[38] Z. Zhuang, V. F. Mitrović, and J. B. Marston, Resistively detected NMR as a probe of the topological nature of conducting edge/surface states, arXiv:2104.00146v1.

[39] B. Venitucci, D. Feinberg, R. Mélin, and B. Douçot, Nonadiabatic Josephson current pumping by chiral microwave irradiation, Phys. Rev. B 97, 195423 (2018). 